\title{
Intestinal-type adenocarcinoma of the sinonasal tract: an update
}

\author{
Vittorio Rampinelli, Marco Ferrari, and Piero Nicolai
}

\begin{abstract}
Purpose of review
Intestinal-type adenocarcinoma (ITAC) is one of the most frequent sinonasal tumors, especially in European countries. The purpose of this article is to review the most recent literature, with special emphasis on biological and genetic profile and treatment guidelines.
\end{abstract}

\begin{abstract}
Recent findings
Results on large series support transnasal endoscopic surgery as the technique of choice in the large majority of patients with ITAC. Adjuvant radiotherapy is recommended in advanced-stage and high-grade lesions. More robust data are required to confirm that early-stage, low-grade lesions can be treated with exclusive surgery. The efficacy of new chemotherapy and biotherapy regimens and the added value of heavy particle radiotherapy are currently under evaluation. With a 5 -year overall survival ranging between 53 and $83 \%$, which is mainly impacted by local recurrences, ITAC requires a more detailed understanding of its biology. Genetic and biological studies have identified alterations in the molecular pathways of EGFR, MET, and H-RAS which might be considered as potential targets for biotherapy.
\end{abstract}

\section{Summary}

Surgery still plays a key role in the treatment of ITAC, but multidisciplinary management is mandatory. Although further validation is needed, the role of nonsurgical treatment strategies is rising, in agreement with the progresses made in the biological profiling of the disease.

\section{Keywords}

intestinal-type adenocarcinoma, sinonasal tumors, transnasal endoscopic surgery

\section{INTRODUCTION}

Intestinal-type adenocarcinoma (ITAC) is one of the most frequent epithelial, nonsquamous cell tumor of the sinonasal tract. The preferential site of origin is commonly identified in the nasoethmoidal complex, but according to the observations of Jankowski et al. [1], the tumor specifically originates from the olfactory cleft. Another typical feature of ITAC is the quite constant association with occupational exposure to wood dust.

In the last decades, a number of refinements in the treatment of ITAC have emerged, including transnasal endoscopic surgery (TES), heavy ion radiation therapy, and chemotherapy as a valuable tool especially in the neoadjuvant setting. Meanwhile, progresses in biological and genetic profiling of ITAC are paving the way toward new target therapies, which need further investigations. The present review is aimed to provide the reader with the main background information on ITAC, together with major advances in the last years.

\section{EPIDEMIOLOGY}

ITAC is a rare tumor with heterogeneous worldwide incidence and sex distribution. In Europe, the agestandardized incidence per 100000 person-years accounts for 0.26 cases in men and 0.04 in women [2"], whereas in the United States, these values are 0.058 in men and 0.034 in women [3]. Accordingly, ITAC is one of the most frequent sinonasal cancers in Europe, whereas it is much rarer in the United States. Similarly, the prevalence in men is remarkably variable, with a men-to-women ratio ranging

Department of Surgical Specialties, Radiological Sciences, and Public Health, Unit of Otorhinolaryngology - Head and Neck Surgery, University of Brescia, Brescia, Italy

Correspondence to Piero Nicolai, MD, Unit of Otorhinolaryngology, Spedali Civili of Brescia, University of Brescia, Piazza Spedali Civili, 1, 25123 Brescia, Italy. Tel: +39 0303995319; fax: +39 030395212; e-mail: pieronicolai@virgilio.it

Curr Opin Otolaryngol Head Neck Surg 2018, 26:000-000 DOI:10.1097/MOO.0000000000000445 


\section{KEY POINTS}

- Sinonasal ITAC is a rare tumor, associated with occupational exposure to wood dust.

- Different histologic subtypes show variable biologic behavior.

- Transnasal endoscopic surgery is the technique of choice in the large majority of patients with ITAC; adjuvant radiotherapy is recommended in advancedstage and high-grade lesions.

- Neoadjuvant chemotherapy with a platinum-based regimen can provide a survival benefit in selected ITACs with functional p53, whereas genetic and biological studies have identified alterations in the molecular pathways of EGFR, MET, and H-RAS, which might be considered as potential targets for biotherapy.

- The efficacy of heavy particle radiotherapy is currently under evaluation.

from 1.07 to 1.39 in the United States up to 6.68 in Italy (Europe) $\left[2^{*}, 3,4\right]$. Median age at diagnosis is 64-68 years, with a peak of incidence at 60-69 years in the United States and 75-84 years in Italy (Europe) $[2 ;, 3]$. Worldwide, the prevalence of ITAC overtime is stable; however, a rising incidence has been observed in some countries (e.g., Denmark) $\left[2^{*}, 5\right]$. The disease is associated with a moderately increased risk of occurrence (odds-ratio: 1.47) and worse prognosis in blacks compared with whites $[3,4]$.

The large majority of ITAC (88\%) can be attributed to occupational exposure [2"]. The most important risk factor is exposure to wood dust [relative risk (RR): 29.4], followed by products in the textile industry (RR: 3.5). Cumulative time of exposure and latency are widely variable, as reported by our group: 2-66 and 11-72 years, respectively [6].

Conversely, exposure to formaldehyde, nickel/ chromium compounds, or asbestos, which has been identified as risk factor for sinonasal cancer, was not confirmed to play a role in the development of adenocarcinoma $[7,8]$.

\section{HISTOPATHOLOGY}

Two main pathological classifications of ITAC are currently used [9]. According to Barnes [10], five categories can be identified: papillary $(18 \%)$, colic $(40 \%)$, solid $(20 \%)$, mucinous $(14 \%)$, and mixed (8\%). Kleinsasser and Schroeder [11] subdivided ITAC into four variants: papillary-tubular cylinder cell, which was further graded from I to III, alveolar goblet, signet-ring cell, and transitional. Histologic diagnosis is relatively simple, and the differential diagnosis includes a secondary localization of colorectal adenocarcinoma and low-grade nonintestinal sinonasal adenocarcinoma [12"'].

ITAC and adenocarcinomas of the intestines show a similar immunophenotypical spectrum: at immunohistochemistry, both stain positive for CK20, CDX-2, villin, and MUC2 [12"-" However, chromogranin A and synaptophysin expression, which can be found in neuroendocrine cells within the tumor, is typical of primary ITAC $\left[12^{-"}, 13\right]$. Positive staining for CK7 supports the diagnosis of sinonasal ITAC over that of intestinal cancer metastasis, although with low sensitivity [14]. Recently, the intestinal transcription and epigenetic factor SATB-2 was identified as an additional marker for ITAC [15]. However, only the integration of pathological and clinical information can differentiate ITAC from metastatic colorectal adenocarcinoma, which accounts for $6.1 \%$ of secondary sinonasal tumors $[12 "=, 16]$.

Differential diagnosis of ITAC from sinonasal nonintestinal adenocarcinomas is guided by immunohistochemistry, as CK20, CDX-2, and villin only stain positive in ITACs [12"'].

\section{CARCINOGENESIS AND GENETICS}

Although the carcinogenesis of ITAC is still far from being fully elucidated, some advances have been made in the last years.

CLU and LGALS4, which are markers of serous cells of the sinonasal lamina propria glands, are upregulated in ITAC, especially in well differentiated forms [17]. Foci of intestinal metaplasia have been described in close vicinity to ITAC (27.5\%), with a high rate of dysplasia (75\%) [18]. TP53 overexpression and mutation are related to wood exposure in both normal mucosa and ITAC $[19,20]$. This evidence suggests that carcinogen(s) contained in wood dust serves as initiation factor(s) for cells of the sinonasal mucosal glands, with tumorigenesis occurring in a multistep fashion similar to other cancer models. Chronic inflammation, which can also be triggered by wood dust, might act as promoting factor; however, no information about the pattern of cytokines involved is available and further research is consequently needed [20]. Moreover, patients affected by ITAC show a higher rate of the CYP1A1 codon 461 polymorphism and SDTM1 null genotype compared with the general population, suggesting that genetic susceptibility is necessary for tumor development, thus explaining why most woodworkers are not affected by ITAC [21].

In recent years, the mutational landscape of ITAC has been better characterized. The spectrum 
of copy number alterations is variable (gains at $1 \mathrm{q}$, $3 q, 5 p, 7,8 q, 11 q, 12 p, 13 q, 20 q$, and 22 and loss at $3 \mathrm{p}, 4,5 \mathrm{q}, 6 \mathrm{q}, 8 \mathrm{p}, 9 \mathrm{p}, 10 \mathrm{q}, 16 \mathrm{q}, 17 \mathrm{p}, 18 \mathrm{q}, 19 \mathrm{p}$, and 21) [20], including chromosomal regions usually involved in most of the epithelial malignancies (gains at $7 \mathrm{p}, 8 \mathrm{q}$, and $20 \mathrm{q}$ and losses at $8 \mathrm{p}$ and $17 p$ ) and others that are typical of ITAC (gains at $3 q, 5 p, 12 p$, and $18 p$ and losses at $4 q, 19 p$, and $22 q$ ) [22].

EGFR copy number gain is frequent (45\%; with protein overexpression in $13-21 \%$ of cases), especially in the colonic subtype [23], which has been attributed to two mechanisms: chromosome seven polysomy and gene amplification [24,25]. Polysomy, which results in aneuploidy, is a clue of chromosomal instability and is associated with poor prognosis $[24,26]$. EGFR protein overexpression is more common in woodworkers $(42.8 \%)$ than in leatherworkers $(9.5 \%)$ and patients without professional exposure (0\%) [25]. Mutations in EGFR (0$5.5 \%)$, K-RAS $(5.5-12 \%)$, and BRAF $(0-5.9 \%)$ are rarely found, are mutually exclusive, and are not associated with EGFR overexpression $[24,27,28]$. This feature differentiates ITAC from colorectal and lung adenocarcinomas, which are commonly associated with EGFR (only lung), K-RAS, and BRAF mutations [22]. The mutation in codon 12 or 13 of K-RAS is thought to be specifically related to wood dust cause [20]. H-RAS is mutated in $16 \%$ of cases and associated with worse prognosis $[20,29]$.

TP53 mutations, promoter methylation, loss of heterozygosity at $17 \mathrm{p} 13$, and $\mathrm{p} 53$ protein overexpression is found in $18-57 \%$ of cases and is related to the subtype of ITAC $[20,30]$. Nonfunctional p53 protein is frequent and associated with reduced chemosensitivity and worse prognosis compared with patients with a functional protein [31,32].

MET is overproduced in $64 \%$ of ITACs; the underlying mechanism of this gain of function is not associated with gene amplification and remains to be determined [33].

Furthermore, a number of other genetic and epigenetic alterations have been described $[20,22$, 30,34-40]. The genes involved and those which are hallmarks of aggressive biological behavior are summarized in Table 1.

Recently, five clusters of gene copy number alterations of ITAC were identified, with a remarkable variability in terms of prognosis; no correlation either with exposure to well known etiological factors or with histological subtypes of ITAC (papillary, colonic, solid, and mucinous) has been found [22]. Rather, the transition from the most differentiated subtype (papillary) to the least differentiated ITAC (mucinous) seems to be related to the total amount of mutations [26].

\begin{tabular}{ll} 
Table 1. The mutational landscape of intestinal-type \\
adenocarcinoma & \\
\hline & $\begin{array}{c}\text { Genes and genetic } \\
\text { alterations associated } \\
\text { with aggressive } \\
\text { benes involved in }\end{array}$ \\
ITAC oncogenesis & Aneuploidy \\
\hline ANXA1 (frequent) & 4q32-ter \\
ANXA2 (rare) & ANXA2 \\
BRAF (rare) & DCC \\
DCC (rare) & H-RAS \\
EGFR gain of function (frequent) & MET \\
EGFR mutation (rare) & MYC \\
HRAS (rare) & PFOU5F1B \\
KRAS (rare) & PTP4A3 \\
MET (frequent) & PTPN1 \\
MYC (rare) & TIMP2 \\
PFOU5F1B (rare) & TIMP3 \\
TP53 (frequent) & TP53 \\
4q32-ter & \\
APC & \\
BCL2 & \\
CCND1 & \\
CDH1 & \\
ESR 1 & \\
GPR124 & \\
ORAOV1 & \\
PTP4A3 & \\
PTPN1 & \\
STK1 1/LKB 1 & \\
IIMP2 & \\
TIMP3 & \\
TRAIL & \\
\end{tabular}

The hallmarks of aggressive biological behavior are summarized in the right column. ITAC, intestinal-type adenocarcinoma.

${ }^{a}$ Advanced stage at diagnosis, high-grade subtypes (solid, mucinous), intracranial invasion, survival outcomes.

\section{DIAGNOSIS AND STAGING}

When a malignant lesion of the sinonasal tract is suspected based on history and endoscopic findings, diagnostic work-up should include morphological imaging followed by biopsy. Contrast-enhanced MRI is the gold standard. It affords precise soft tissue characterization with high-contrast resolution, which discriminates neoplastic tissue from nasal secretions and inflammatory changes, and defines any possible critical extension (dural/transdural involvement, perineural spread, intraorbital extension, etc.). Computed tomography (CT) provides additional information on tissue density (i.e., calcifications or bony structures), but is frequently unnecessary in the hands of an expert radiologist.

On MRI, the signal intensity of adenocarcinomas varies according to their mucin content, cellularity, and presence of hemorrhagic-necrotic areas. Mucin-producing adenocarcinomas usually show spontaneous hyperintensity on T2-weighted images and gradual enhancement on contrast-enhanced 
T1-weighted sequences, whereas adenocarcinomas without mucin production show isointensity to hypointensity on T2-weighted images. However, the imaging features of adenocarcinomas are often indistinguishable from those of other sinonasal cancers [41].

In view of the key role of histology in guiding treatment, it is important that biopsy is accurately obtained and histologic slides are analyzed by a pathologist with specific expertise. In fact, the rarity of sinonasal tumors and the wide range of histologies explain the high rate of diagnostic discrepancies detected after pathologic revision by expert pathologists $[42,43]$. Recently, our group reported a 16.9\% rate of discrepancies when comparing pretreatment biopsy and definitive histologic examination in a cohort of 77 patients affected by nasoethmoidal malignancies entirely managed in a tertiary referral center. Biopsy reliability rate was primarily influenced by histology, with adenocarcinoma being less frequently associated with diagnostic error compared with other rarer entities. However, the site, pattern of growth, radiological features of the lesion, and clinical-anatomical patient-related variables (comorbidities, pain-threshold) should be considered when choosing the surgical procedure for biopsy. In unfavorable conditions, biopsy under sedation and local anesthesia or diagnostic debulking under general anesthesia should be preferred over outpatient sampling of the tumor [44].

Sinonasal ITAC has a low tendency to lymphatic dissemination: nodal metastases are rare, accounting for $2.4-8.0 \%$ at presentation and $1.8-2.4 \%$ during follow-up, being distinctive of an aggressive behavior of the tumor $[6,45,46]$. Cervical metastases mostly occur at level II and must be confirmed with ultrasound with or without fine-needle aspiration cytology. Retrolateropharyngeal nodal metastases are likely underestimated, but can be easily detected with MRI or CT. Distant metastases are identified in $1.7 \%$ of cases at presentation and $12.8 \%$ of cases during follow-up [47]. At PET, ITAC shows a highstandard uptake value, which is second only to sinonasal undifferentiated carcinoma [48]. Consequently, primary regional and distant staging as well as follow-up may benefit from PET, especially in advanced-stage and high-grade ITACs [6]. Total body CT associated with bone scintigraphy can be a reasonable alternative.

\section{TREATMENT}

Surgery represents the mainstay of treatment for ITAC, usually followed by adjuvant intensity-modulated radiotherapy (IMRT). However, in the era of histology-driven multimodal treatment strategies for sinonasal cancer [49], the role of chemotherapy and adrotherapy is gaining prominence.

\section{Surgery}

Surgical planning is guided by both preoperative imaging and intraoperative findings. Whatever the approach, the aim is to perform radical resection using frozen sections to map the surgical margins.

In the last 15 years, TES has emerged as the surgical approach of choice for most sinonasal cancer located in the nasoethmoidal complex $\left[6,50^{-}, 51\right]$. External or combined approaches (craniofacial and cranioendoscopic resection, respectively) still have a role in selected cases (i.e., massive brain infiltration, supraorbital dural extension, nasal bones/facial invasion, massive involvement of the lacrimal system, and/or orbital content) [49].

Endoscopic resection with transnasal craniectomy is indicated when the tumor is in contact or crosses the anterior skull base, to provide an adequate margin of resection. After anterior skull base resection is performed, a watertight reconstruction of the dura is required to avoid cerebrospinal fluid leak. Although the use of pedicled flaps has dramatically decreased this occurrence [52], they are rarely available due to either direct tumor infiltration or the need to sacrifice the vascular pedicle. On the other hand, three-layer reconstruction with iliotibial tract grafts has been validated as a reliable and safe technique for anterior skull base defects $[53,54]$. Morbidity of endoscopic surgery for sinonasal malignancies is limited, with excellent outcomes in terms of quality of life, hospitalization time, morbidity, and mortality compared with traditional external approaches $[53,55]$.

The concept of 'field of cancerization' as the result of wood dust (or other factors) inhalation led to consider bilateral resection of the ethmoidal box a paradigm in surgery of ITAC, regardless of the extent of primary tumor [21]. Against this philosophy, some authors reported good oncological outcomes even with unilateral resection applied to strictly lateralized lesions [56]. As shown in a recent report on olfactory neuroblastoma, a unilateral resection technique can have the advantage of sparing some olfactory function in approximately half of patients, although undergoing radiotherapy [57]. However, as data in the literature are not robust enough to draw definitive conclusions, more stringent posttreatment surveillance is mandatory when a limited resection is performed.

In view of the low rate of cervical lymph node metastases at presentation and during follow-up, elective neck treatment is not indicated $[47,58]$. 


\section{Radiotherapy}

Photon radiation therapy is usually administrated as adjuvant treatment after surgery in high-grade and/ or advanced-stage tumors [6]. Recent reports seem to confirm that early-stage (T1-2), low-grade (papillary/colonic) ITACs can be adequately treated with radical surgery alone $[59,60]$.

Given the proximity to highly radiosensitive anatomical structures (i.e., orbital content, optic nerves, brain), the use of IMRT is strongly recommended over conventional radiation techniques, with the intent to minimize treatment toxicity [61].

Recently, the role of heavy ion therapy, such as proton beam radiation therapy, is also emerging: adjuvant proton beam radiation for sinonasal cancers was reported to provide a local control superior to conventional radiotherapy or IMRT $[62,63]$. This is likely due to the possibility to better sharp the dose gradient in view of the physical features of heavy particles, thus optimizing the balance between the need for high dose on the tumor bed and low dose on neighboring noble structures. Likewise, carbon-ion radiation therapy is a promising strategy for sinonasal adenocarcinoma [64]. In case of nonresectable tumors, radiotherapy with photons and/or neutrons, possibly associated with chemotherapy, provides a valuable 5 -year overall survival (OS); however, specific data on ITAC are lacking [65].

Finally, perioperative image-adapted brachytherapy has also been proposed for sinonasal cancer (including ITAC), with 3-year OS of 72\% [66].

\section{Chemotherapy}

The only data available on chemotherapy for sinonasal ITAC are based on the Italian experience at the 'Istituto Nazionale dei Tumori' in Milan [31,32]. Neoadjuvant chemotherapy with cisplatin, 5-fluorouracil, and leucovorin (PFL) resulted in a pathological complete response (CR) in up to $40 \%$ of cases. The probability to obtain a CR to chemotherapy was significantly higher in ITACs with functional p53 ( $58 \%$ of cases), including both the wild-type and the mutated protein with function preservation or gain. Based on these data, neoadjuvant chemotherapy with PFL scheme is indicated only for ITACs bearing functional p53 protein [67]. Apart from this isolated experience, the role of chemotherapy in the neoadjuvant or adjuvant setting in the management of ITAC has not been systematically investigated.

Local application of fluorouracil after debulking surgery has also been proposed as primary treatment for ITAC [68], but the Dutch experience has not been reproduced on a large scale.

\section{Biotherapy}

Although no clinical data are available on biotherapy for ITAC, the biological profile resulting from several preclinical studies and the availability of an immortalized tumor cell line encourages the employment of target therapies in the management of this tumor $[20,69]$. By virtue of a low incidence of EGFR, K-RAS, and BRAF mutations and high rate of EGFR copy number gain, the mutational profile of ITAC seems particularly fitting to anti-EGFR drugs $[27,28]$. Likewise, the high rate of MET mutation (64\%) makes MET inhibitors another interesting option [20,33]. The subgroup of patients with $\mathrm{H}$ RAS mutation (16\%) may benefit from the administration of MAPK/ERK pathway inhibitors, alone or combined with inhibitors of the cyclin-dependent kinase-4/6 [20,70]. Specific studies are warranted to explore the real benefit of these drugs in a clinical setting.

\section{PROGNOSTIC FACTORS AND ONCOLOGIC OUTCOMES}

Oncologic outcomes are influenced by several factors, such as patient characteristics or biological features of the tumor. Age higher than 75 years, male sex, and black race have been identified as adverse prognostic factors [71]. From a histopathologic perspective, mucinous and solid subtypes are associated with poor prognosis $\left[47,50^{-}, 71\right]$. At presentation, locally advanced tumors, nodal or distant metastases, and sphenoidal, orbital, dural, or brain invasion were demonstrated as negative prognosticators $\left[6,33,50^{-}, 60,71-74\right]$. High proliferation index and several other biological markers (Table 1) are significantly associated with worse prognosis [75].

Surgery with free margins and overall adequacy of primary management are of utmost importance, as positive margins, local failure, and inadequate previous treatments are independently associated with poor prognosis $\left[6,33,50^{-}, 73,76\right]$. Adjuvant radiation therapy has an independent positive effect on survival $[59,71]$.

In the largest published series, 5-year overall, disease-specific, and disease-free survivals are 53-83, $82-83$, and $62-74 \%$, respectively $\left[6,46,50^{-}, 77,78\right]$. Open surgery (i.e., craniofacial resection) is associated with higher morbidity and a nonnegligible mortality rate $[45,51,55]$. The recurrence rate after definitive treatment ranges from 17.6 to $49.6 \%$, with local, regional, and distant relapse accounting for $16.0-38.2,1.8-2.4$, and $6.5-12.8 \%$, respectively $\left[6,46,47,50^{\mathbf{n}}, 78\right]$. Accordingly, postoperative surveillance should mainly focus on the primary site, by including endoscopic examination and MRI (or 
contrast-enhanced CT) at 3-month and 6-month intervals for the first 24 months, respectively; beyond this period, both outpatient and imaging evaluations can be performed every 6 months until year 5 and subsequently once a year [79-81], in view of the possibility for ITAC to develop late local recurrences. Similarly to primary staging, PET/CT imaging can be considered for high-grade/high-stage ITACs also during follow-up. It is worth mentioning that recurrent ITAC has a remarkably aggressive behavior, which is similar to that of high-grade histologies (e.g., mucosal melanomas, sinonasal undifferentiated carcinomas, sinonasal neuroendocrine carcinomas, sarcomas) [82].

\section{CONCLUSION}

Sinonasal ITAC is a rare tumor with variable biologic behavior in relation to the histologic subtype. TES has emerged as an appropriate primary treatment for most ITACs. Adjuvant IMRT is recommended in advanced lesions and high-grade tumors. The possibility to avoid any adjuvant treatment in earlystage, radically excised, low-grade lesions should be further explored. Neoadjuvant chemotherapy with a platinum-based regimen can provide a survival benefit in selected ITACs with functional p53.

Although the biology of ITAC is far from being fully elucidated, recent advances in understanding the carcinogenetic mechanisms and genetic alterations involved in the tumor may contribute to defining a role for biotherapy in the therapeutic armamentarium.

\section{Acknowledgements}

We would like to thank Dr Davide Mattavelli and Dr Alberto Schreiber for the precious assistance provided for the preparation of the study.

\section{Financial support and sponsorship}

None.

\section{Conflicts of interest}

There are no conflicts of interest.

\section{REFERENCES AND RECOMMENDED}

\section{READING}

Papers of particular interest, published within the annual period of review, have been highlighted as:

- of special interest

-1. of outstanding interest

1. Jankowski R, Georgel T, Vignaud JM, et al. Endoscopic surgery reveals that woodworkers' adenocarcinomas originate in the olfactory cleft. Rhinology 2007; 45:308-314.

2. Binazzi $A$, Corfiati M, Di Marzio D, et al. Sinonasal cancer in the Italian national

- surveillance system: epidemiology, occupation, and public health implications. Am J Ind Med 2017; doi: 10.1002/ajim.22789. [Epub ahead of print] The article describes the clinical characteristics and occupational exposures of a cohort of more than 1500 sinonasal cancer cases.
3. Kilic S, Samarrai R, Kilic SS, et al. Incidence and survival of sinonasal adenocarcinoma by site and histologic subtype. Acta Otolaryngol 2017; 1-7; doi: 10.1080/00016489.2017.1401229. [Epub ahead of print]

4. D'Aguillo CM, Kanumuri VV, Khan MN, et al. Demographics and survival trends of sinonasal adenocarcinoma from 1973 to 2019. Int Forum Allergy Rhinol 2014; 4:771-776.

5. Youlden DR, Cramb SM, Peters S, et al. International comparisons of the incidence and mortality of sinonasal cancer. Cancer Epidemiol 2013; 37:770-779.

6. Nicolai $P$, Schreiber A, Bolzoni Villaret A, et al. Intestinal type adenocarcinoma of the ethmoid: outcomes of a treatment regimen based on endoscopic surgery with or without radiotherapy. Head Neck 2016; 38(Suppl 1): E996-E1003.

7. Binazzi A, Ferrante $P$, Marinaccio A. Occupational exposure and sinonasal cancer: a systematic review and meta-analysis. BMC Cancer 2015; 15:49.

8. Andersson M, Selin F, Jarvholm B. Asbestos exposure and the risk of sinonasal cancer. Occup Med (Lond) 2016; 66:326-331.

9. Stelow EB, Franchi A, Wenig BM. Intestinal-type adenocarcinoma. In: ElNaggar AK, Chan JCK, Grandis JR, et al., editors. WHO classification of head and neck tumours. Lyon: International Agency for Research on Cancer (IARC); 2017

10. Barnes L. Intestinal-type adenocarcinoma of the nasal cavity and paranasal sinuses. Am J Surg Pathol 1986; 10:192-202.

11. Kleinsasser $O$, Schroeder HG. The pathology and clinical picture of adenocarcinoma of the nose after wood dust exposure. Strahlenther Onkol 1989; 165:437-440.

12. Leivo I. Intestinal-type adenocarcinoma: classification, immunophenotype,

- molecular features and differential diagnosis. Head Neck Pathol 2017; $11: 295-300$.

The article provides an update on current histopathologic classification of intestinal-type adenocarcinoma (ITAC), immunophenotypic properties, recent advances in molecular pathologic features, and differential diagnostic considerations.

13. Projetti F, Serrano E, Vergez $S$, et al. Is neuroendocrine differentiation useful to discriminate primary sinonasal intestinal-type adenocarcinomas from metastatic colorectal adenocarcinomas? J Clin Pathol 2015; 68: 79-82.

14. Franchi A, Massi D, Palomba A, et al. CDX-2, cytokeratin 7 and cytokeratin 20 immunohistochemical expression in the differential diagnosis of primary adenocarcinomas of the sinonasal tract. Virchows Arch 2004; 445: 63-67.

15. Skalova A, Sar A, Laco J, et al. The role of SATB2 as a diagnostic marker of sinonasal intestinal-type adenocarcinoma. Appl Immunohistochem Mol Morphol 2016; doi: 10.1097/PAI.0000000000000388. [Epub ahead of print]

16. Mills SE, Fechner RE, Cantrell RW. Aggressive sinonasal lesion resembling normal intestinal mucosa. Am J Surg Pathol 1982; 6:803-809.

17. Tripodi D, Quemener S, Renaudin K, et al. Gene expression profiling in sinonasal adenocarcinoma. BMC Med Genomics 2009; 2:65.

18. Franchi $A$, Palomba $A$, Miligi $L$, et al. Intestinal metaplasia of the sinonasal mucosa adjacent to intestinal-type adenocarcinoma. A morphologic, immunohistochemical, and molecular study. Virchows Arch 2015; 466:161-168.

19. Perez-Escuredo J, Martinez JG, Vivanco B, et al. Wood dust-related mutational profile of TP53 in intestinal-type sinonasal adenocarcinoma. Hum Pathol 2012; 43:1894-1901.

20. Hoeben $A$, van de Winkel $L$, Hoebers $F$, et al. Intestinal-type sinonasal adenocarcinomas: the road to molecular diagnosis and personalized treatment. Head Neck 2016; 38:1564-1570.

21. Cantu G, Solero CL, Mariani L, et al. Intestinal type adenocarcinoma of the ethmoid sinus in wood and leather workers: a retrospective study of 153 cases. Head Neck 2011; 33:535-542.

22. Lopez-Hernandez A, Perez-Escuredo J, Vivanco B, et al. Genomic profiling of intestinal-type sinonasal adenocarcinoma reveals subgroups of patients with distinct clinical outcomes. Head Neck 2017; doi: 10.1002/hed.24941. [Epub ahead of print]

23. Szablewski V, Solassol J, Poizat F, et al. EGFR expression and KRAS and BRAF mutational status in intestinal-type sinonasal adenocarcinoma. Int J Mol Sci 2013; 14:5170-5181.

24. Projetti $F$, Durand $K$, Chaunavel $A$, et al. Epidermal growth factor receptor expression and KRAS and BRAF mutations: study of 39 sinonasal intestinaltype adenocarcinomas. Hum Pathol 2013; 44:2116-2125.

25. Franchi $A$, Fondi $C$, Paglierani $M$, et al. Epidermal growth factor receptor expression and gene copy number in sinonasal intestinal type adenocarcinoma. Oral Oncol 2009; 45:835-838.

26. Hermsen MA, Llorente JL, Perez-Escuredo J, et al. Genome-wide analysis of genetic changes in intestinal-type sinonasal adenocarcinoma. Head Neck 2009; 31:290-297.

27. Garcia-Inclan C, Lopez F, Perez-Escuredo J, et al. EGFR status and KRAS/ BRAF mutations in intestinal-type sinonasal adenocarcinomas. Cell Oncol 2012; 35:443-450.

28. Franchi A, Innocenti DR, Palomba A, et al. Low prevalence of K-RAS, EGF-R and BRAF mutations in sinonasal adenocarcinomas. Implications for antiEGFR treatments. Pathol Oncol Res 2014; 20:571-579. 
29. Perez $P$, Dominguez $O$, Gonzalez $S$, et al. ras Gene mutations in ethmoid sinus adenocarcinoma: prognostic implications. Cancer 1999; 86:255-264.

30. Re M, Magliulo G, Tarchini $P$, et al. p53 and BCL-2 over-expression inversely correlates with histological differentiation in occupational ethmoidal intestinaltype sinonasal adenocarcinoma. Int J Immunopathol Pharmacol 2011; 24:603-609.

31. Licitra L, Suardi S, Bossi P, et al. Prediction of TP53 status for primary cisplatin, fluorouracil, and leucovorin chemotherapy in ethmoid sinus intestinal-type adenocarcinoma. J Clin Oncol 2004; 22:4901-4906.

32. Bossi P, Perrone F, Miceli R, et al. Tp53 status as guide for the management of ethmoid sinus intestinal-type adenocarcinoma. Oral Oncol 2013; 49: 413-419.

33. Projetti F, Mesturoux L, Coulibaly B, et al. Study of MET protein levels and MET gene copy number in 72 sinonasal intestinal-type adenocarcinomas. Head Neck 2015; 37:1563-1568.

34. Franchi A, Palomba A, Fondi C, et al. Immunohistochemical investigation of tumorigenic pathways in sinonasal intestinal-type adenocarcinoma. A tissue microarray analysis of 62 cases. Histopathology 2011; 59:98-105.

35. Perez-Escuredo J, Lopez-Hernandez A, Costales M, et al. Recurrent DNA copy number alterations in intestinal-type sinonasal adenocarcinoma. Rhinology $2016 ; 54: 278-286$.

36. Chiang JM, Chen TC. A Peutz-Jeghers syndrome family associated with sinonasal adenocarcinoma: 28 years follow up report. Fam Cancer 2017; 16:555-560.

37. Costales M, Lopez-Hernandez A, Garcia-Inclan C, et al. Gene methylation profiling in sinonasal adenocarcinoma and squamous cell carcinoma. Otolaryngol Head Neck Surg 2016; 155:808-815.

38. Diaz-Molina JP, Llorente JL, Vivanco B, et al. Wnt-pathway activation in intestinal-type sinonasal adenocarcinoma. Rhinology 2011; 49:593-599.

39. Re M, Santarelli A, Mascitti M, et al. Trail overexpression inversely correlates with histological differentiation in intestinal-type sinonasal adenocarcinoma. Int J Surg Oncol 2013; 2013:203873.

40. Rodrigo JP, Garcia-Pedrero JM, Llorente JL, et al. Down-regulation of annexin $\mathrm{A} 1$ and $\mathrm{A} 2$ protein expression in intestinal-type sinonasal adenocarcinomas. Hum Pathol 2011; 42:88-94.

41. Kawaguchi $M$, Kato $H$, Tomita $H$, et al. Imaging characteristics of malignant sinonasal tumors. J Clin Med 2017; 6:116.

42. Kronz JD, Westra WH. The role of second opinion pathology in the management of lesions of the head and neck. Curr Opin Otolaryngol Head Neck Surg 2005; 13:81-84.

43. Mehrad M, Chernock RD, El-Mofty SK, Lewis JS Jr. Diagnostic discrepancies in mandatory slide review of extradepartmental head and neck cases: experience at a large academic center. Arch Pathol Lab Med 2015; 139:1539-1545.

44. Schreiber A, Rampinelli V, Ferrari M, et al. Diagnostic reliability of pretreatment biopsy in malignant naso-ethmoidal tumors: a retrospective study on 78 cases. Laryngoscope 2018; doi: 10.1002/lary.27077. [Epub ahead of print]

45. Mortuaire G, Leroy X, Vandenhende-Szymanski C, et al. Comparison of endoscopic and external resections for sinonasal instestinal-type adenocarcinoma. Eur Arch Otorhinolaryngol 2016; 273:4343-4350.

46. Vergez $S$, du Mayne MD, Coste A, et al. Multicenter study to assess endoscopic resection of 159 sinonasal adenocarcinomas. Ann Surg Oncol 2014; 21:1384-1390.

47. Donhuijsen K, Kollecker I, Petersen P, et al. Metastatic behaviour of sinonasal adenocarcinomas of the intestinal type (ITAC). Eur Arch Otorhinolaryngol 2016; 273:649-654.

48. Felix-Ravelo M, Bey A, Arous F, et al. Relationship between (18)FDG-PET and different types of sinonasal malignancies. Acta Otolaryngol 2017; 137:191-195.

49. Castelnuovo $P$, Turri-Zanoni $M$, Battaglia $P$, et al. Sinonasal malignancies of anterior skull base: histology-driven treatment strategies. Otolaryngol Clin North Am 2016; 49:183-200.

50. Camp S, Van Gerven L, Poorten VV, et al. Long-term follow-up of 123 patients

- with adenocarcinoma of the sinonasal tract treated with endoscopic resection and postoperative radiation therapy. Head Neck 2016; 38:294-300.

This is a large and homogeneous series of patients affected by ITAC of the sinonasal tract, showing that in well selected patients and with appropriate use of adjuvant therapy, endoscopic resection of sinonasal adenocarcinoma results in good oncologic outcomes.

51. Meccariello G, Deganello A, Choussy O, et al. Endoscopic nasal versus open approach for the management of sinonasal adenocarcinoma: a pooled-analysis of 1826 patients. Head Neck 2016; 38(Suppl 1):E2267-E2274.

52. Klatt-Cromwell CN, Thorp BD, Del Signore AG, et al. Reconstruction of skull base defects. Otolaryngol Clin North Am 2016; 49:107-117.

53. Mattavelli $D$, Schreiber $A$, Villaret $A B$, et al. Complications and donor site morbidity of 3-layer reconstruction with iliotibial tract of the anterior skull base: retrospective analysis of 186 patients. Head Neck 2018; 40:63-69.

54. Mattavelli D, Schreiber A, Ferrari $M$, et al. Three-layer reconstruction with iliotibial tract after endoscopic resection of sinonasal tumors. World Neurosurg 2017; 101:486-492.

55. Castelnuovo $P$, Lepera $D$, Turri-Zanoni $M$, et al. Quality of life following endoscopic endonasal resection of anterior skull base cancers. J Neurosurg 2013; 119:1401-1409.
56. Van Gerven L, Jorissen M, Nuyts $S$, et al. Long-term follow-up of 44 patients with adenocarcinoma of the nasal cavity and sinuses primarily treated with endoscopic resection followed by radiotherapy. Head Neck 2011; 33: 898-904.

57. Tajudeen BA, Adappa ND, Kuan EC, et al. Smell preservation following endoscopic unilateral resection of esthesioneuroblastoma: a multiinstitutional experience. Int Forum Allergy Rhinol 2016; 6:1047-1050.

58. Mirghani H, Hartl D, Mortuaire G, et al. Nodal recurrence of sinonasal cancer: does the risk of cervical relapse justify a prophylactic neck treatment? Oral Oncol 2013; 49:374-380.

59. Turri-Zanoni $M$, Battaglia $P$, Lambertoni $A$, et al. Treatment strategies for primary early-stage sinonasal adenocarcinoma: a retrospective bi-institutional case-control study. J Surg Oncol 2015; 112:561-567.

60. Choussy O, Ferron C, Vedrine PO, et al. Role of radiotherapy in the treatment of nasoethmoidal adenocarcinoma. Arch Otolaryngol Head Neck Surg 2010; 136:143-146.

61. Askoxylakis $V$, Hegenbarth $P$, Timke $C$, et al. Intensity modulated radiation therapy (IMRT) for sinonasal tumors: a single center long-term clinical analysis. Radiat Oncol 2016; 11:17.

62. Patel SH, Wang Z, Wong WW, et al. Charged particle therapy versus photon therapy for paranasal sinus and nasal cavity malignant diseases: a systematic review and meta-analysis. Lancet Oncol 2014; 15:1027-1038.

63. Dagan R, Bryant C, Li Z, et al. Outcomes of sinonasal cancer treated with proton therapy. Int J Radiat Oncol Biol Phys 2016; 95:377-385.

64. Koto $M$, Hasegawa $A$, Takagi $R$, et al. Feasibility of carbon ion radiotherapy for locally advanced sinonasal adenocarcinoma. Radiother Oncol 2014; 113:60-65.

65. Chopra S, Kamdar DP, Cohen DS, et al. Outcomes of nonsurgical management of locally advanced carcinomas of the sinonasal cavity. Laryngoscope $2017 ; 127: 855-861$.

66. Teudt IU, Meyer JE, Ritter M, et al. Perioperative image-adapted brachytherapy for the treatment of paranasal sinus and nasal cavity malignancies. Brachytherapy $2014 ; 13: 178-186$.

67. Bossi P, Saba NF, Vermorken JB, et al. The role of systemic therapy in the management of sinonasal cancer: a critical review. Cancer Treat Rev 2015; $41: 836-843$.

68. Knegt PP, Ah-See KW, vd Velden LA, Kerrebijn J. Adenocarcinoma of the ethmoidal sinus complex: surgical debulking and topical fluorouracil may be the optimal treatment. Arch Otolaryngol Head Neck Surg 2001; 127: $141-146$.

69. Perez-Escuredo J, Garcia Martinez J, Garcia-Inclan C, et al. Establishment and genetic characterization of an immortal tumor cell line derived from intestinaltype sinonasal adenocarcinoma. Cell Oncol 2011; 34:23-31.

70. Yadav V, Burke TF, Huber L, et al. The CDK4/6 inhibitor LY2835219 overcomes vemurafenib resistance resulting from MAPK reactivation and cyclin D1 upregulation. Mol Cancer Ther 2014; 13:2253-2263.

71. Chen MM, Roman SA, Sosa JA, Judson BL. Predictors of survival in sinonasal adenocarcinoma. J Neurol Surg B Skull Base 2015; 76:208-213.

72. Howard DJ, Lund VJ, Wei WI. Craniofacial resection for tumors of the nasal cavity and paranasal sinuses: a 25-year experience. Head Neck 2006; 28:867-873.

73. Suarez C, Llorente JL, Fernandez De Leon R, et al. Prognostic factors in sinonasal tumors involving the anterior skull base. Head Neck 2004; 26:136-144.

74. Fiaux-Camous $D$, Chevret $S$, Oker $N$, et al. Prognostic value of the seventh AJCC/UICC TNM classification of intestinal-type ethmoid adenocarcinoma: systematic review and risk prediction model. Head Neck 2017; 39:668-678.

75. Antognoni $P$, Turri-Zanoni $M$, Gottardo $S$, et al. Endoscopic resection followed by adjuvant radiotherapy for sinonasal intestinal-type adenocarcinoma: retrospective analysis of 30 consecutive patients. Head Neck 2015; 37:677-684.

76. Hanna E, DeMonte F, Ibrahim S, et al. Endoscopic resection of sinonasal cancers with and without craniotomy: oncologic results. Arch Otolaryngol Head Neck Surg 2009; 135:1219-1224.

77. Lund V, Howard DJ, Wei WI. Endoscopic resection of malignant tumors of the nose and sinuses. Am J Rhinol 2007; 21:89-94.

78. Bogaerts S, Vander Poorten V, Nuyts S, et al. Results of endoscopic resection followed by radiotherapy for primarily diagnosed adenocarcinomas of the paranasal sinuses. Head Neck 2008; 30:728-736.

79. Parasher AK, Kuan EC, John MAS, et al. What is the appropriate timing for endoscopic and radiographic surveillance following treatment for sinonasal malignancies? Laryngoscope 2017; doi: 10.1002/lary.27013. [Epub ahead of print]

80. Workman AD, Palmer JN, Adappa ND. Posttreatment surveillance for sinonasal malignancy. Curr Opin Otolaryngol Head Neck Surg 2017; 25:86-92.

81. Maroldi $R$, Ravanelli $M$, Farina $D$, et al. Posttreatment evaluation of paranasal sinuses after treatment of sinonasal neoplasms. Neuroimaging Clin N Am 2015; 25:667-685.

82. Kaplan DJ, Kim JH, Wang E, Snyderman C. Prognostic indicators for salvage surgery of recurrent sinonasal malignancy. Otolaryngol Head Neck Surg 2016; 154:104-112. 